\title{
Tenecteplase versus Alteplase
}

Fragestellung: Ist die Gabe von Tenecteplase in der Behandlung des akuten ischämischen Insults der Gabe von Alteplase überlegen?

Hintergrund: Die seit vielen Jahren in Deutschland und Europa zugelassene Standardtherapie des akuten ischämischen Insults im Zeitfenster von 4,5 Stunden ist die systemische Gabe von Alteplase mit einem initialen Bolus, gefolgt von einer Infusion, wobei die Dosis gewichtsadaptiert wird. Der Nutzen dieser Therapie ist durch mehrere große randomisierte Studien und Register belegt. Tenecteplase wird im Gegensatz zu Alteplase nur als Bolus gegeben und hat eine längere Halbwertszeit. In einer australischen Phase-II-Studie zeigten Patienten, die mit Tenecteplase behandelt wurden, einen besseren klinischen Outcome und eine höhere Reperfusionsrate als mit Alteplase behandelte Patienten. Eine norwegische Arbeitsgruppe wollte jetzt in einer großen Phase-III-Studie die Überlegenheit von Tenecteplase belegen.

Patienten und Methodik: Die randomisierte, offene Überlegenheitsstudie erfolgte in 13 Stroke Units in Norwegen, die Adjudizierung der Endpunkte verblindet. Eingeschlossen wurden Patienten innerhalb eines Zeitfensters von 4,5 Stunden nach Beginn der Symptomatik. Sie erhielten entweder 0,4 mg/kg Körpergewicht Tenecteplase mit einer Maximaldosis von $40 \mathrm{mg}$ oder Alteplase $0,9 \mathrm{mg} / \mathrm{kg}$ Körpergewicht mit einer Maximaldosis von $90 \mathrm{mg}$, wobei $10 \%$ als Bolus und $90 \%$ über eine Stunde als Infusion gegeben wurden. Der primäre Endpunkt war ein Wert auf der modifizierten Rankin-Skala (mRS) von 0 bis 3 nach drei Monaten.

Ergebnisse: Die Studie schloss 1.107 Patienten ein, von denen 549 Tenecteplase und 551 Alteplase erhielten. Das mittlere Alter der Patienten betrug 77 Jahre; der mediane Wert auf der NIHSS lag bei 4 Punkten. Je $20 \%$ der Patienten hatten eine Makroangiopathie oder eine Kardioembolie. Im Mittel vergingen 33 Minuten zwischen Krankenhausaufnahme und Beginn der Thrombolyse. Den primären Endpunkt erreichten 354 Patienten (64\%) in der Tenecteplase- und 345 Patienten (63\%) in der Alteplase-Gruppe. Die Mortalität betrug in beiden Gruppen nach drei Monaten jeweils $5 \%$. Die Rate schwerwiegender Nebenwirkungen und Blutungskomplikationen war in beiden Gruppen mit $26 \%$ ebenfalls identisch.

Logallo N, Novotny V, Assmus J et al. Tenecteplase versus alteplase for management of acute ischaemic stroke (NOR-TEST): a phase 3, randomised, open-label, blinded endpoint trial. Lancet Neurol 2017; 16: $781-8$
Schlussfolgerungen: In einer großen randomisierten Studie zeigte sich kein Unterschied in der therapeutischen Wirkung von Tenecteplase und Alteplase bei Patienten mit akutem ischämischem Insult, die im Zeitfenster von 4,5 Stunden behandelt wurden.

\section{- Kommentar von Hans Christoph Diener, Essen}

\section{Alteplase bleibt Standard}

Entgegen den Erwartungen der Studiengruppe zeigte Tenecteplase keine therapeutische Überlegenheit im Vergleich zu Alteplase. Alle Endpunkte inklusive der Sicherheitsendpunkte waren in beiden Therapiegruppen identisch. Obwohl Tenecteplase leichter zu applizieren ist (als Bolus) wird es in Deutschland bis auf weiteres bei einer Therapie mit Alteplase bleiben. Die Studie hatte darüber hinaus das Problem, dass der Löwenanteil der Patienten nur leichte Schlaganfälle hatte. Ob Tenecteplase bei Patienten mit schweren Schlaganfällen möglicherweise überlegen ist, ist aus der vorliegenden Studie nicht zu erschließen. 